\section{Changes in the trend of hydrocephalus subtypes in infants}

TO THE EDITOR: We read with great interest the paper by Radic et al. ${ }^{2}$ (Radic JAE, Vincer M, McNeely PD: Temporal trends of intraventricular hemorrhage of prematurity in Nova Scotia from 1993 to 2012. J Neurosurg Pediatr 15:573-579, June 2015) regarding the chronological outline of the rate of intraventricular hemorrhage (IVH) and hydrocephalus in premature infants. The authors performed a population-based study on 1334 very preterm infants born in a period of 20 years and found that the percentage of very preterm infants with IVH Grade 3 or 4 has significantly increased over time. Moreover, the incidence of posthemorrhagic hydrocephalus (PHH) and shunt surgery in infants with Grade 3 or $4 \mathrm{IVH}$ has increased meaningfully ( $\mathrm{p}=0.001$ and $\mathrm{p}=0.011$, respectively), but the proportion of patients with $\mathrm{PHH}$ receiving a shunt has not changed over time.

Over this longitudinal study, though the percentage of successfully resuscitated very preterm infants with IVH of any grade did not change significantly, both 7-day and 28-day mortality in successfully resuscitated very preterm infants did significantly decrease. ${ }^{2}$ So, this increasing trend of high-grade IVH and PHH may be partially attributed to improving survival. However, the association between PHH and time and between shunting and time remains significant even when controlling for improved survival, suggesting that other factors are probably at play. The variables found to be independently associated with Grade 3 or 4 IVH included antenatal steroid use, infant cardiopulmonary resuscitation, dopamine use, maternal fever during labor, and death in the first 7 days.

We would like to describe our own experience with hydrocephalus and IVH of prematurity at the Children's Hospital Medical Center in Tehran, Iran, over a period of 6 years, from 2008 to 2014 . We found a rising trend in shunt surgeries for $\mathrm{PHH}$ at our center, which is consistent with the results of the Radic et al. survey. At the same time, we encountered a decreased rate of myelomeningocele (MMC) patients and so a decrease in shunt procedures related to associated hydrocephalus in MMC patients. Our center is a tertiary care hospital, and the pattern of referral has not changed during the last 14 years; therefore, this trend cannot be related to different patterns of the referral of sick patients to this center.

In 2008, among those who underwent their first proce- dures for hydrocephalus (not including revision surgeries), $18.7 \%$ were cases of $\mathrm{PHH}$ and $20.4 \%$ were MMC-associated hydrocephalus (MMC-H), with a PHH/MMC-H ratio of 0.89 . This ratio was elevated to 1.3 in 2009 and continued growing (with slight fluctuation) to 2.12 and 2.25 in the years 2013 and 2014, respectively. The parallel trends of a decrease in MMC-H and an increase in PHH can be attributed to improved prenatal screening and care for neural tube anomalies, relatively high rate of preterm deliveries (compared to Western countries), ${ }^{1}$ and better survival of preterm infants. In recent years, folate supplementation, prenatal screening protocol, and early diagnosis and termination of pregnancies with a diagnosis of MMC have led to decreased rates of MMC-H. On the other hand, the availability of more neonatal intensive care units during recent years has been associated with better care of very ill premature neonates and thus may increase the number of premature neonates. Moreover, the growing rate and success of in vitro fertilization in our country in recent years may have a role in increasing the rate of multiple pregnancies, in turn leading to more premature births. And nowadays, as deduced by Radic et al., more premature neonates survive to infancy, which can be another factor for the ascending incidence of PHH and related shunt implantations.

So, the changes in the distribution of hydrocephalus subtypes in infants can be a reflection of changes in other disciplines, such as perinatology, neonatal intensive care, and even infertility treatments.

Zohreh Habibi, MD

Farideh Nejat, MD, MPH

Tehran University of Medical Science, Tehran, Iran

\section{References}

1. Alijahan R, Hazrati S, Mirzarahimi M, Pourfarzi F, Ahmadi Hadi P: Prevalence and risk factors associated with preterm birth in Ardabil, Iran. Iran J Reprod Med 12:47-56, 2014

2. Radic JAE, Vincer M, McNeely PD: Temporal trends of intraventricular hemorrhage of prematurity in Nova Scotia from 1993 to 2012. J Neurosurg Pediatr 15:573-579, 2015

\section{Disclosures}

The authors report no conflict of interest.

\section{Response}

We thank Drs. Habibi and Nejat for their letter and for 
sharing their experience with IVH in prematurity, $\mathrm{PHH}$, and hydrocephalus associated with MMC at the Children's Hospital Medical Center in Tehran, Iran, over a 6-year period. Consistent with the Nova Scotia experience, they have observed an increase in shunt operations for PHH. They have also noticed a simultaneous decrease in shunt operations for MMC patients, probably as a result of fewer admissions due to MMC. They identify that the increasing incidence of PHH and the decreasing incidence of MMC$\mathrm{H}$ at their center may be attributable to the evolution in prenatal care, increased incidence of prematurity, and improvements in the survival of preterm infants.

Certain causes of neonatal hydrocephalus are strongly associated with population health and are at least partially preventable through modifying known risk factors. For example, folic acid fortification reduces the risk of MMC$\mathrm{H}$, and improving socioeconomic conditions reduces the risk of prematurity and thus reduces the risk of IVH in prematurity and $\mathrm{PHH}$. What makes our paper's findings interesting is that, independent of confounders, major IVH risk factors, and improved survival, the incidence of IVH and PHH appears to be increasing in the Nova Scotia population. Given the often-disabling lifelong impact of severe IVH and $\mathrm{PHH}$, we have a public health duty to learn whether there are any significant modifiable causes for this increase and then make appropriate changes in care to reduce the risk of IVH and PHH. It will be the purview of future research to determine whether modifying the potential causal factors identified by our analysis will improve outcomes in this patient population.

Julia A. E. Radic, MD, MPH University of British Columbia, Vancouver, BC, Canada

Michael Vincer, MD

Neonatal-Perinatal Medicine, Dalhousie University, Halifax, NS, Canada

P. Daniel McNeely, MD

IWK Health Centre, Halifax, NS, Canada

Dalhousie University, Halifax, NS, Canada

\section{INCLUDE WHEN CITING}

Published online December 11, 2015; DOI: 10.3171/2015.7.PEDS15402.

cAANS, 2016 